\title{
Effect of Time-strengthening Static Friction on Earthquake Recurrence
}

\author{
Jeen-Hwa Wang \\ Institute of Earth Sciences, Academia Sinica, jhwang@earth.sinica.edu.tw \\ Article history: received November 3, 2019; accepted September 15, 2020
}

\begin{abstract}
The effect of time-strengthening static friction on earthquake recurrence is numerically studied based on the normalized equation of motion of a one-body spring-slider model with thermal-pressurized slip-weakening friction having the characteristic distance, $\mathrm{U}_{\mathfrak{c}}$, which is in terms of static friction coefficient, $\mu_{f}$. Considering the time-strengthening static friction coefficient, $\mu_{f}(t)=\mu_{f o}+B \times \log (t)$, we assume $U_{c}=U_{c o} /(1+B \times \log (t))$. Simulation results exhibit that $T_{R}, \tau_{D}, D$, and $V_{m}$, which represent the recurrence time of two events, the duration time of slip of an event, the final slip of an event, and the peak value of particle velocity of an event, respectively, are all similar for five values of $U_{c o}$ when $B<0.01$ and clearly different when $B \geq 0.01$. In general, $V_{m}, D$, and $T_{R}$ increase with $B$; and $\tau_{D}$ slightly decreases with increasing $B$ and increases with $U_{c o}$. An increase in $D$ is particularly remarkable when $U_{c o}>0.3$. The earthquake recurrence is almost periodic for small $U_{c o}$; while the degree of periodicity decreases when either $U_{c o}$ or $B$ increases. Either the time-predictable model or the slip-predictable model can approximately interpret the simulated earthquake recurrences with small $U_{c o}$ and $\operatorname{small} B$, yet not for those with large $U_{c o}$ and large $B$. Simulation results exhibit that time-strengthening static friction produce an opposite effect on earthquake recurrence from the time-widening slip zone.
\end{abstract}

Keywords: Earthquake recurrence; Final slip; Rise time; One-body spring-slider model; Thermalpressurized slip-weakening friction

\section{Introduction}

Earthquake recurrence, which represents the repetitive occurrences of events on a fault during a long time period, is an important factor in assessing seismic hazards. The time variation in slip and particle velocity during a seismic cycle is shown in Figure 1 where $T_{R}, \tau_{D}, D$, and $V_{m}$, represent, respectively, the recurrence (also denoted by repeat or inter-event) time of two events, the duration time of slip of an event, the final slip of an event, and the peak value of particle velocity of an event. For a perfectly periodic earthquake recurrence system, the four parameters may be constants in the entire time history because all model parameters do not vary with time. On the other hand, they could vary from cycle to cycle when one of model parameters is time-varying. The major factors in controlling $T_{R}$ include the plate moving speed and the geometry of the rupture zone [Sykes and Quittmeyer, 1981]. Based on the elastic rebound theory proposed by Reid [1910], Schwartz and Coppersmith [1984] assumed that an earthquake 


\section{Jeen-Hwa Wang}

happens when the tectonic shear stress on a fault is higher than a critical level, which depends on the physical conditions of the fault and tectonic loading. In their studies earthquakes may happen regularly because of homogeneous physical properties on a fault and constant tectonic loading.

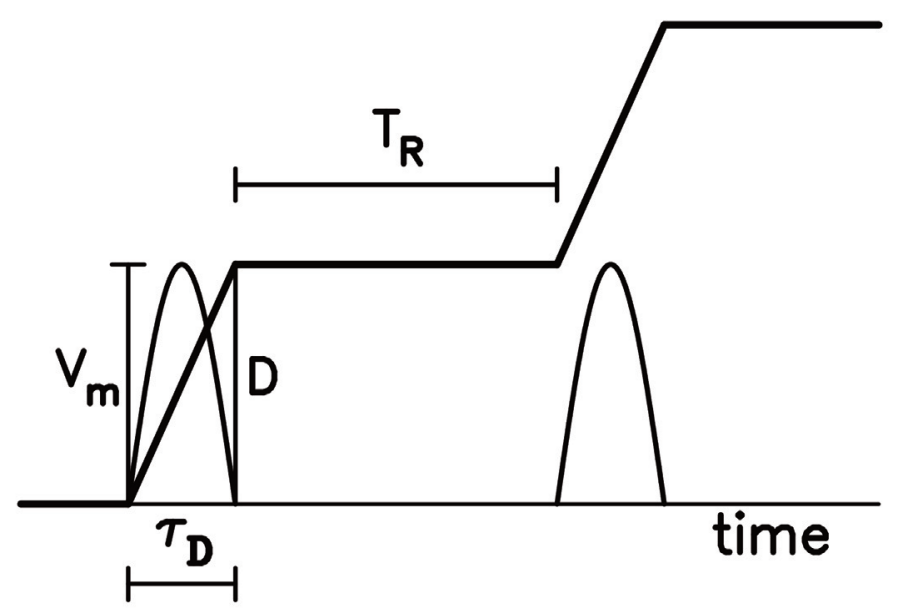

Figure 1. A general pattern of time variations in slip (thick solid line) and particle velocity (thin solid curve) during a seismic cycle: $T_{R}=$ the recurrence time or the inter-event time of two events in a seismic cycle; $\tau_{D}=$ the duration time of slip of an event; $D=$ the final slip of an event; and $V_{m}=$ the peak particle velocity of an event [after Wang, 2018].

Shimazaki and Nakata [1980] proposed three simple phenomenological models to interpret earthquake recurrences. The time variations of cumulative slip related to the three models are shown in Figure 2. In their models, tectonic loading on a fault increases constantly with time and failure of the fault is controlled by a critical stress level, $\sigma_{c}$. After failure, the fault stops when the stress drops to a base stress level, $\sigma_{b}$. The physical conditions of the three models are: (1) constant $\sigma_{\mathcal{G}} \sigma_{b}$, and $\Delta \sigma$ for the perfectly periodic model (see Figure 2a); (2) constant $\sigma_{c}$,

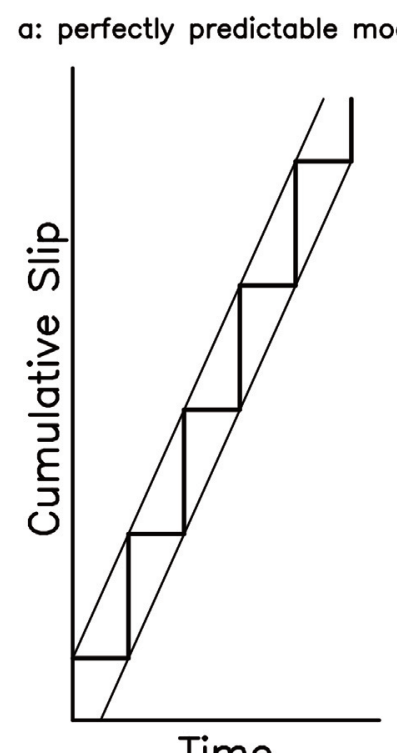

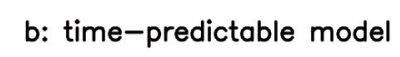

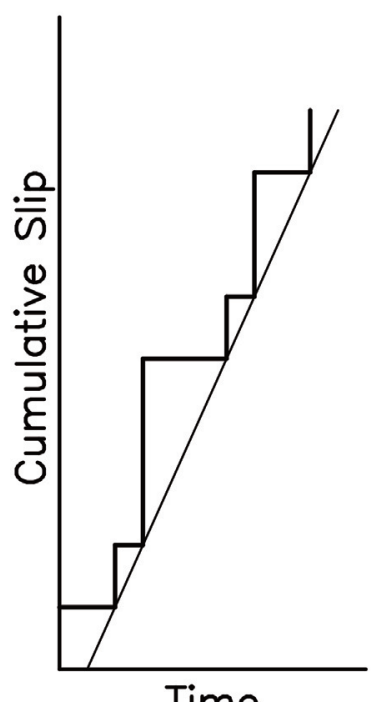

Time

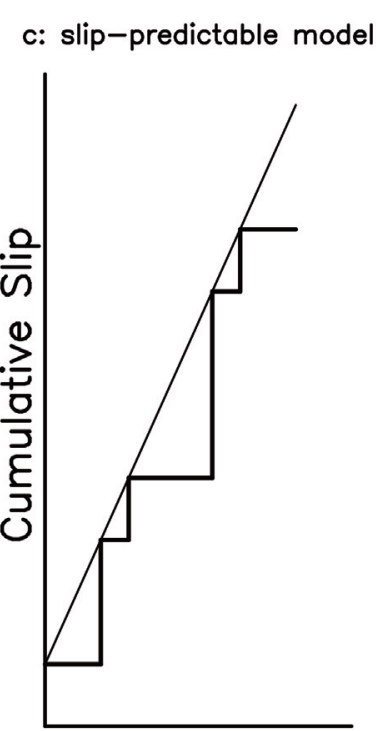

Time

Figure 2. The earthquake recurrence models (the horizontal axis for time and the vertical axis for cumulative slip): (a) perfectly predictable model; (b) time-predictable model; and (c) slip-predictable model [after Wang, 2019]. 
variable $\sigma_{b}$, and variable $\Delta \sigma$ for the time-predictable model (see Figure 2b); and (3) variable $\sigma_{c}$, constant $\sigma_{b}$, and variable $\Delta \sigma$ for the slip-predictable model (see Figure 2c). For the first model, both $T_{R}$ and $D$ of next earthquake can be predicted from the values of $T_{R}$ or $D$ of previous ones. For the second model, $T_{R}$ of next event can be predicted from the values of $D$ of previous ones. For the third model, $D$ of next event can be predicted from the values of $T_{R}$ of previous ones.

However, the three models have been debated for a long time. Some dicussions are given below. Ando [1975] suggested that the second model worked for post-1707 events, yet not for pre-1707 ones in the Nankai trough, Japan. Wang [2005, 2019] assumed that the second model could describe the earthquakes occurred on the Chelungpu fault, Taiwan in the past 1900 years. For the Parkfield earthquake sequence, Bakun and McEvilly [1984] took different models; while Murray and Segall [2002] considered the failure of the second model. From laboratory results, Rubinstein et al. [2012] assumed the failure of the time- and slip-predictable models for earthquakes.

Numerous physical models, including the kinetic, crack, and dynamical spring-sider models have been developed for approaching fault dynamics. Unfortunately, up to date there is not a comprehensive model which can completely describe fault dynamics. Two major factors in controlling fault dynamics and earthquake ruptures [Bizzarri, 2009; Wang, 2017b, 2018] are friction [Nur, 1978; Belardinelli and Belardinelli, 1996] and viscosity [Jeffreys, 1942; Spray, 1983; Wang, 2007]. Earthquake recurrence has been simulated based on different models [Bizzarri 2012a, b; Franović et al., 2016; Wang, 2018]. A detailed description about the studies based on the spring-slider model [Burridge and Knopoff, 1967] and its simplified forms with various friction laws can be seen in Wang [2018]. Among the physical models to approach earthquake faults, the one-body spring-slider model is the simplest and useful one to represent a single fault.

Wang [2018] numerically simulated earthquake recurrence by using the normalized equation of motion of a onebody spring-sider model with thermal-pressurized slip-weakening friction and viscosity. The main parameters are the normalized characteristic displacement, $U_{c}$, of the friction law and the normalized damping coefficient, $\eta$, which is used to represent viscosity. His simulation results show that $T_{R}$ increases with decreasing $U_{c}$ or increasing $\eta$; $D$ and $\tau_{D}$ decrease with increasing $\eta$; and $\tau_{D}$ increases with $U_{c}$. The time- and slip-predictable model can describe the temporal variation in cumulative slip. Considering the wear process, he assumed that the thickness of slip zone, $h$, is an important parameter influencing $T_{R}$ and $D$ and also related $h$ to the cumulated slip $S=\sum D$ in the form: $h=C S$ ( $C=$ a dimensionless increasing rate of $h$ with $S$ from a cycle to next one). $U_{c}$ is a function of $h$ and thus depends on cumulated slip, $\Sigma U$, with an increasing rate of $C$. His simulation results reveal that the wear process influences the recurrence of events and the effect increases with $C$ when $C>0.0001$. Although viscosity can strengthen the effect due to wear process it cannot change the pattern of earthquake recurrence. Both $T_{R}$ and $D$ decrease when the fault becomes more mature, thus suggesting that it is more difficult to produce large earthquakes along a fault when it is more mature with longer $h$. Neither the time-predictable model nor the slip-predictable one can describe the temporal variation in cumulative slip of earthquakes under the wear process with large $C$. Due to a lack of long-term variation in $\eta$ and viscosity can only strengthens the effect and cannot change the pattern of earthquake recurrence as mentioned in Wang [2018]. Hence, the viscous effect is not included in this study.

In addition to the wear process, static friction (represented by the static friction coefficient, $\mu_{f}$ ) also affects $U_{c}$, thus being able to change the pattern of earthquake recurrence. The value of $\mu_{f}$ could be time-dependent. In the followings, we will investigate the effect on earthquake recurrence caused by time-strengthening static friction coefficient based on a one-body spring-slider model. From numerical simulations based on this model in the presence of thermal-pressurized friction and viscosity, we can obtain significant information of frictional and viscous effects on earthquake recurrence along a single fault.

\section{One-body model and numerical methodology}

Figure 3 displays the one-body spring-slider model, in which $m, K, \sigma_{n}, F, u, v(=d u / d t), v_{p}$, and $u_{0}=v_{p} t$ denote, respectively, the mass of the slider, the stiffness (or spring constant) of the leaf spring, the normal stress, the frictional force between the slider and the moving plate, the displacement of the slider, the velocity of the slider, the plate moving speed, and the equilibrium location of the slider. The frictional force $F$ (with the static value $F_{0}$ ) is usually a function of $u$ or $v$. A driving force, $K v_{p} t$, caused by the moving plate through the leaf spring pulls the slider to move. The equation of motion is: 


\section{Jeen-Hwa Wang}

$m d^{2} u / d t^{2}=-K\left(u-u_{0}\right)-F(u, v)$

When $K v_{p} t \geq F_{O}, F$ drops from static to dynamic frictional force and then pushes the slider to move.

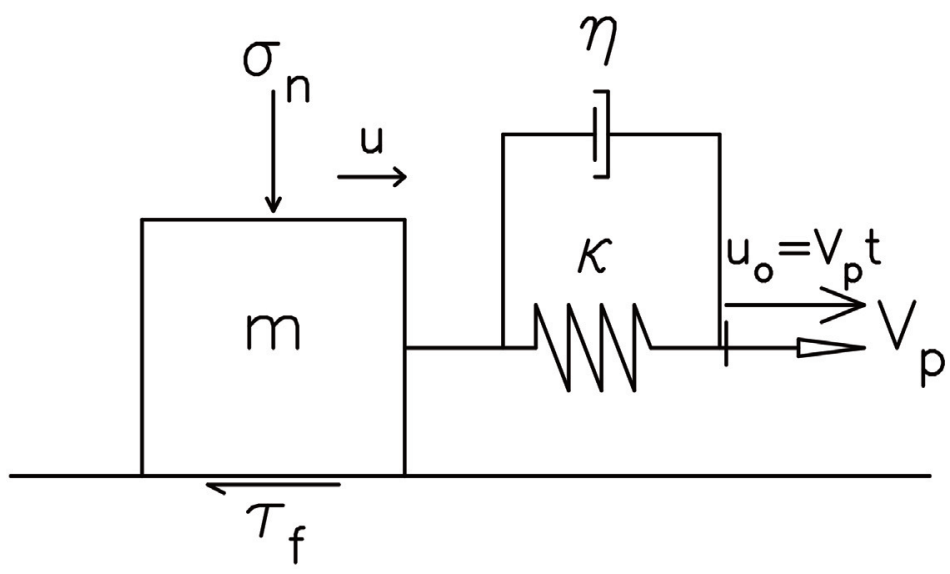

Figure 3. One-body spring-slider model. In the figure, $t, m, K, V_{\mathrm{p}}, N, F u$, and $u_{o}$ denote, respectively, the time, the mass of the slider, the spring constant, the driving velocity, the normal force, the frictional force, displacement of the slider, and the equilibrium location of the slider [after Wang, 2016].

Numerous models have been proposed to describe $F(u, v)$ [Wang, 2016]. One of them is proposed based on thermal pressurization (abbreviated as TP below) which is caused by the combined effect from temperature and fluids in a fault zone and thus yields a shear stress (resistance) on the fault plane [Rice, 2006; Wang, 2009, 2011, 2016, 2017a, b, 2018]. The 1-D TP model proposed by Rice [2006] has two end-member models: one is the adiabatic-undrained-deformation (AUD) model and the other the slip-on-a-plane (SOP) model. Since the characteristic distance of the SOP model is not associated with the wear process, the model is not used in this study. The AUD model is related to a homogeneous simple strain $\varepsilon$ at a constant normal stress $\sigma_{n}$ on a spatial scale of the sheared layer. Its shear stress-slip function, $\tau(u)$, is [Rice, 2006]: $\tau(u)=\mu_{f}\left(\sigma_{n}-p_{o}\right) \exp \left(-u / u_{c}\right)$ where $p_{o}$ is the pore fluid pressure. Clearly, $\tau(u)$ decreases exponentially with increasing $u$. The characteristic displacement is $u_{c}=\rho_{f} C_{v} h / \mu_{f} \Lambda$, where $\rho_{f}, C_{v}, h, \mu_{f}$, and $\Lambda$ are, respectively, the fluid density, heat capacity, the thickness, frictional strength, and the undrained pressurization factor of the fault zone. The detailed description about the model parameters can see Rice [2006].

Based on the AUD model, Wang [2009] first took a simplified slip-weakening friction law (denoted by the TP law hereafter) in the following form:

$$
F(u)=F_{o} \exp \left(-u / u_{c}\right)
$$

which is only dependent on a slip and independent on $v$. Clearly, $F(u)$ at $u=0$ is $F_{0}$. The plot of $F(u)$ versus $u$ when $u_{c}=0.1,0.3,0.5,0.7$, and $0.9 \mathrm{~m}$ and $F_{o}=1 \mathrm{~N} / \mathrm{m}^{2}$ is displayed in Figure $4 . F(u)$ decreases with increasing $u$ and its decreasing rate, $\gamma$, decreases with increasing $u_{c}$. The force drop decreases with increasing $u_{c}$ for the same final slip.

Substituting Equation (2) into Equation (1) leads to

$$
m d^{2} u / d t^{2}=-K\left(u-v_{p} t\right)-F_{o} \exp \left(-u / u_{c}\right)
$$

The easily conduct numerical computations, Equation (3) is normalized based on the normalization parameters: $D_{O}=F_{O} / K, \omega_{O}=(K / m)^{1 / 2}, \tau=\omega_{O} t, U=u / D_{O}$, and $U_{C}=u_{C} / D_{O}$. The normalized velocity, acceleration, and driving velocity are, respectively, $V=d U / d \tau=\left[F_{O} /(m K)^{1 / 2}\right]^{-1} d u / d t, A=d^{2} U / d \tau^{2}=\left(F_{O} / m\right)^{-1} d^{2} u / d t^{2}$, and $V_{p}=v_{p} /\left(D_{O} \omega_{O}\right)$. Note that all normalization parameters are dimensionless. Define $\Omega=\omega / \omega_{0}$ to be the dimensionless angular 


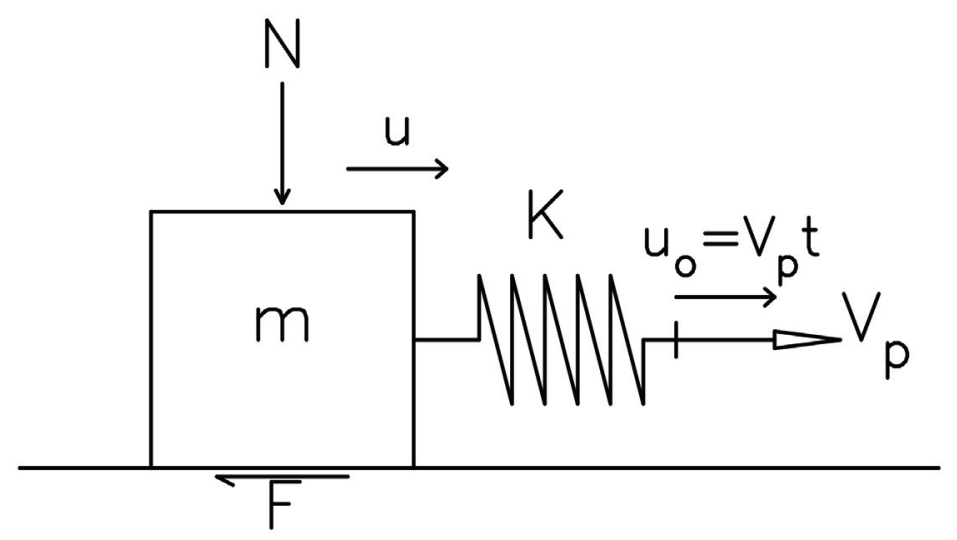

Figure 4. The plots of $F(u)=F_{o} \exp \left(-u / u_{c}\right)$ versus $u$ when $u_{c}=0.1,0.3,0.5,0.7$, and $0.9 \mathrm{~m}$ when $F_{o}=1 \mathrm{Nt} / \mathrm{m}^{2}$ [after Wang, 2016].

frequency. This makes the phase $\omega t$ be $\Omega \tau$. Substituting all normalization parameters into Equation (3) leads to

$$
d^{2} U / d \tau^{2}=-U-\exp \left(-U / U_{C}\right)+V_{p} \tau
$$

To re-write Equation (4) as two first-order differential equations by using two new parameters, i.e., $y_{1}=U$ and $y_{2}=d U / d \tau$, we have

$$
\begin{gathered}
d y_{1} / d \tau=y_{2} \\
d y_{2} / d \tau=-y_{1}-\exp \left(-y_{1} / U_{c}\right)+V_{p} \tau .
\end{gathered}
$$

Equation (5) can be numerically solved by using the fourth-order Runge-Kutta method [Press et al., 1986].

The general values of these model parameters are evaluated by Wang [2018] and also briefly explained below. The values of $D_{o}$ are usually several meters and $\omega_{o}$ ranges from $0.1 \mathrm{~Hz}$ to few $\mathrm{Hz}$ [Wang, 2016]. This leads to that $D_{o} \omega_{o}$ has an order of magnitude of $1 \mathrm{~m} / \mathrm{s}$. The value of $V_{p}$ is much smaller than 1 because of $v_{p} \approx 10^{-10} \mathrm{~m} / \mathrm{s}$. Wang [2018] assumed that since the value of $V_{p}$ mainly influences the recurrence time, $T_{R}$, between two events and can only make a very small influence on the pattern of time variations in velocities and displacements of events. In order to study long-term earthquake recurrence, there must be numerous modeled events with clear and visualized time functions of displacements and velocities for an event in the computational time period. If $V_{p}=10^{-10}$ is considered, $T_{R}$ is very long and thus $\tau_{D}$ is much shorter than $T_{R}$. This makes the time function of an event displayed in the long-term temporal variation in slip looks like just a step function for the displacements and an impulse for the velocities. Hence, in order to get fine visualization a larger value of $V_{p} \tau$ is necessary. The value of $V_{p} \tau$ is usually very small during an event and cannot influence the rupture because of a very tiny value of $V_{p}$. From numerical tests Wang [2018] stressed that when $V_{p}>10^{-2}$, the value of $V_{p} \tau$ is not small during an event and can influence the rupture. Hence, he took $V_{p}=10^{-2}$ for numerical simulations. This value is also used in this study. Note that backward slip is not allowed in the simulations, because of common behavior of forward faulting.

Some researchers [e.g., Bizzarri, 2012a, b; and Franović et al., 2016] suggested time-varying earthquake recurrence or non-perfectly periodic seismic cycle. Wang [2018] considered the role of time-dependent $U_{c}$ on the phenomenon based on the TP model. From $U_{c}=u_{c} / D_{o}$, we have $U_{c}=\rho_{f} C_{V} h / \mu_{f} D_{0} \Lambda$ which $U_{c}$ is controlled by six factors, i.e., $\rho_{f}, C_{V}, h, \mu_{f}, D_{o}$, and $\Lambda$ (in term of $\rho_{f}$ and $n$ ). Wang [2018] deeply discussed the effects of the six model parameters on $U_{c}$. He assumed that $C_{V}$ and $D_{o}$ are minor in affecting $U_{c}$; while time-dependent $\rho_{f}$ and $\Lambda$ are important in influencing $U_{c}$ and thus on earthquake recurrence. But, he did not take $\rho_{f}$ and $\Lambda$ into account because of rare observed data and theoretical analyses. He considered the effect of $h$ on earthquake recurrence. 


\section{Jeen-Hwa Wang}

He assumed that $U_{c}$ varies with cumulative slip in the following way: $U_{c}=U_{c o}+C \sum U$ where $U_{c o}$ is the initial value of $U_{c}$ and $\Sigma U$ is the sum of final displacements of cycles. His results remarkably reveal the effect of timewidening $h$ on earthquake recurrence.

In addition to $h$, the time-varying static friction coefficient, $\mu_{f}$, of the slipping zone which can also influences earthquake recurrence is considered in this study. The $\mu_{f}$ is influenced by several factors including humidity, temperature, sliding velocity, strain rate, normal stress, thermally activated rheology etc. [Marone, 1998; Rice, 2006], and thus can change with time [Sibson, 1992; Rice, 2006]. Hirose and Bystricky [2007] observed that serpentine dehydration and subsequent fluid pressurization due to co-seismic frictional heating may reduce $\mu_{f}$ and thus promote further weakening in a fault zone. The pore fluid pressure exists in wet rocks, yet not in dry rocks. From the laboratory experiments for Tennessee sandstone, Shimamoto and Logan [1981] observed a decrease in $\mu_{f}$ with increasing clay content in the fault-zone gouge and the decreasing rate depends on the types of minerals in the gouge. From the laboratory experiments for Westerly granite, Marone et al. [1990] found an increase in $\mu_{f}$ with gouge thickness. From the laboratory experiments, several researchers [e.g., Ohnaka, 1996; Kamer and Marone, 1998; and Marone 1998] found an increase in $\mu_{f}$ with the logarithmic value of hold time of an experiment. Time-dependence of clay content is not clear. Although the gouge thickness increases with the width of the slipping zone, not any clear relationship between them has been given. Hence, according to abovementioned time-dependence of $\mu_{f}$, a form: $\mu_{f}(t)=1+B \times \log (t)$, where $B$ is an increasing rate of $\mu_{f}$ with $\log (t)$, is considered. The estimated value of $B$ from experimental data is $\sim 0.07$ from Ohnaka [1996], 0.007-0.013 from Kamer and Marone [1998], and 0.007-0.011 from Marone [1998]. Its value could vary for different rocks. The positive dependence of $\mu_{f}$ on time exhibits time-strengthening static friction. Since $u_{c}$ is inversely proportional to $\mu_{f}$ and $U_{c}=u_{C} / D_{o}, U_{c}$ is inversely related to $B$. Here, we assume that $U_{c}$ varies with time in the following manner: $U_{c}=U_{c o} /[1+B \times \log (\tau)]$.

A phase portrait, which is a plot of a physical quantity, $Y$, versus another, $X$, i.e., $Y=f(X)$, is commonly used to represent nonlinear behavior of a dynamical system [Thompson and Stewart, 1986]. The intersection point between $f(X)$ and the bisection line of $Y=X$, is defined as the fixed point, that is, $f(X)=X$. If $f(X)$ is continuously differentiable in an open domain near a fixed point $x_{f}$ and $\left|f^{\prime}\left(X_{f}\right)\right|<1$, attraction can appear at the fixed point. In other words, for any value of $X$ in the domain which is close enough to $X_{f}$, the iterated function sequences, i.e., $X, f(X), f^{2}(X), f^{3}(X), \ldots$, converges to $X_{f}$. Chaos can also be generated at some attractors. The details can be seen in Thompson and Stewart [1986]. In this study, there are three physical quantities, i.e., the acceleration, velocity, and displacement of the slider. The phase portrait can be made from a pair of any two of the three quantities. Here, I take the velocity and displacement to form a phase portrait. Hence, " $Y$ " represents $V / V_{\max }$ and " $X$ " denotes $U / U_{\max }$ in Figures 5-9.

\section{Simulation results}

Here, we investigate the effect of time-strengthening static friction on the patterns of temporal variations in cumulated slip, we must first understand the time-dependent function of $u_{f}$. Simulation results for four values of $B$ are shown in Figures 5-9: (a) for $B=0.001$; (b) for $B=0.010$; (c) for $B=0.050$; and (d) for $B=0.100$. The initial values of $U_{c o}$ are: 0.1 for Figure 5, 0.3 for Figure 6, 0.5 for Figure 7, 0.7 for Figure 8, and 0.9 for Figure 9. Note that the value $U_{c}$ varies with time due to time-varying $\mu_{f}$. In order to plot the temporal variations in normalized velocities and displacements, the maximum of the values of $V_{m}$ of all cycles and the total displacement, $\Sigma U$, in the final cycle to be $V_{\max }$ and $U_{\max }$, respectively, to normalize the velocities and displacements of all modelled events during the computational time in each figure. In each figure, the $V_{\max }$ and $U_{\max }$ appear in the panel (a) of a figure and $V_{\max }$ and $U_{\max }$ decrease from case (a) to case (d). The temporal variations in $V / V_{\max }$ (displayed by thin solid lines) and cumulative slip $\Sigma U / U_{\max }$ (displayed by solid lines) are displayed in the left-handed-side panels. The normalization scales to plot the temporal variations in slip and velocity are $V_{\max }$ for the velocities and the final value of $\Sigma U / U_{\max }$ for the displacements in the computational time. Hence, the upper bound scale is set to be one for the two temporal variations. The phase portraits displayed in the right-handed-side panels of each figure are the plots of $V / V_{\max }$ versus $U / U_{\max }$. In each figure, the value of $U_{c o}$ increases from panel (a) to panel (d); and for each panel of a figure, the value of $U_{c o}$ increases with time. 
Static Friction and Earthquake Recurrence
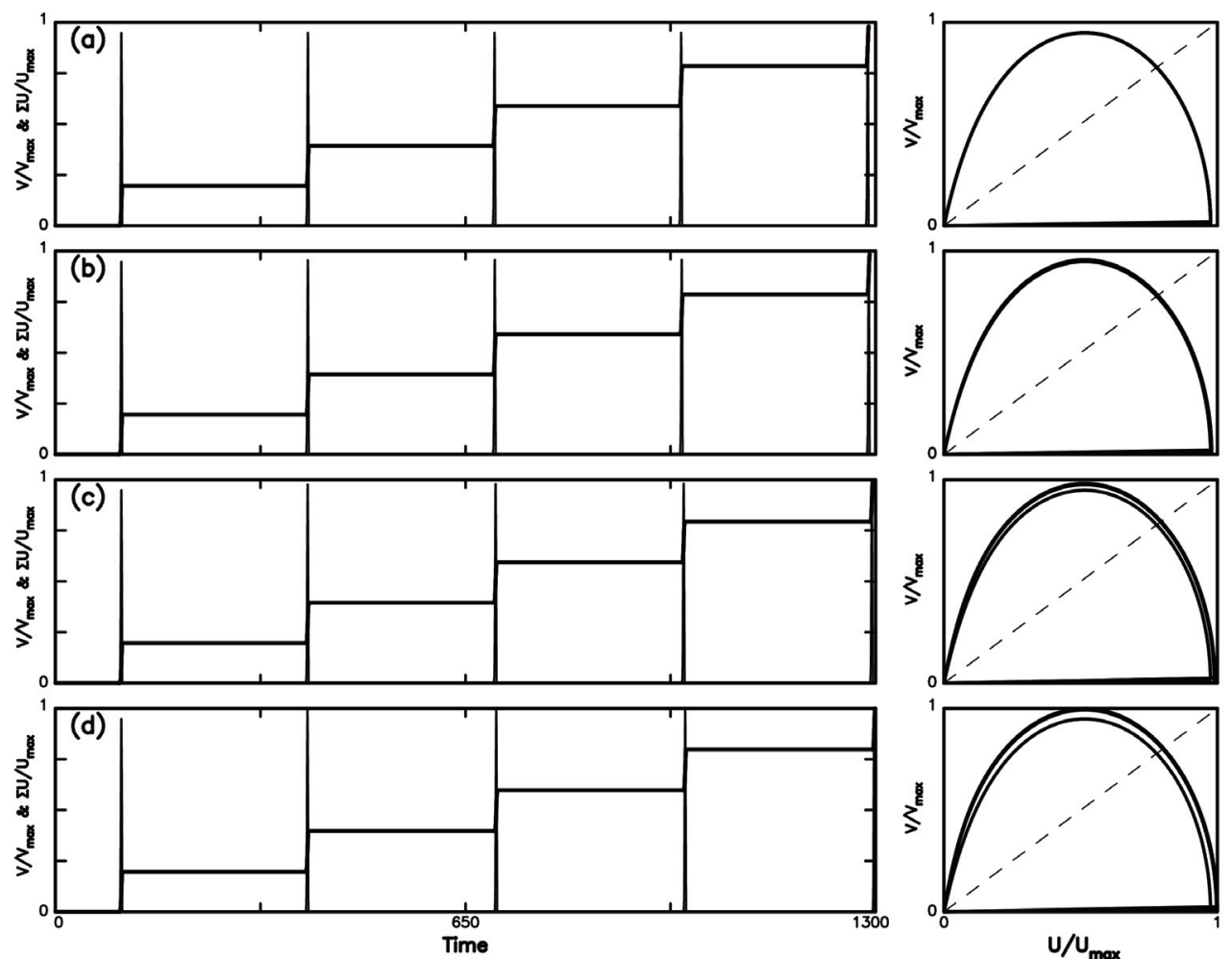

Figure 5. The time variations in $V / V_{\max }$ (thin solid line) and cumulative slip $\Sigma U / U_{\max }$ (solid line) and the phase portrait of $V / V_{\max }$ versus $U / U_{\max }$ (solid line) for four values of $B$ : (a) for $B=0.001$; (b) for $B=0.010$; (c) for $B=0.050$; and (d) for $B=0.100$ when $U_{c o}=0.1$.
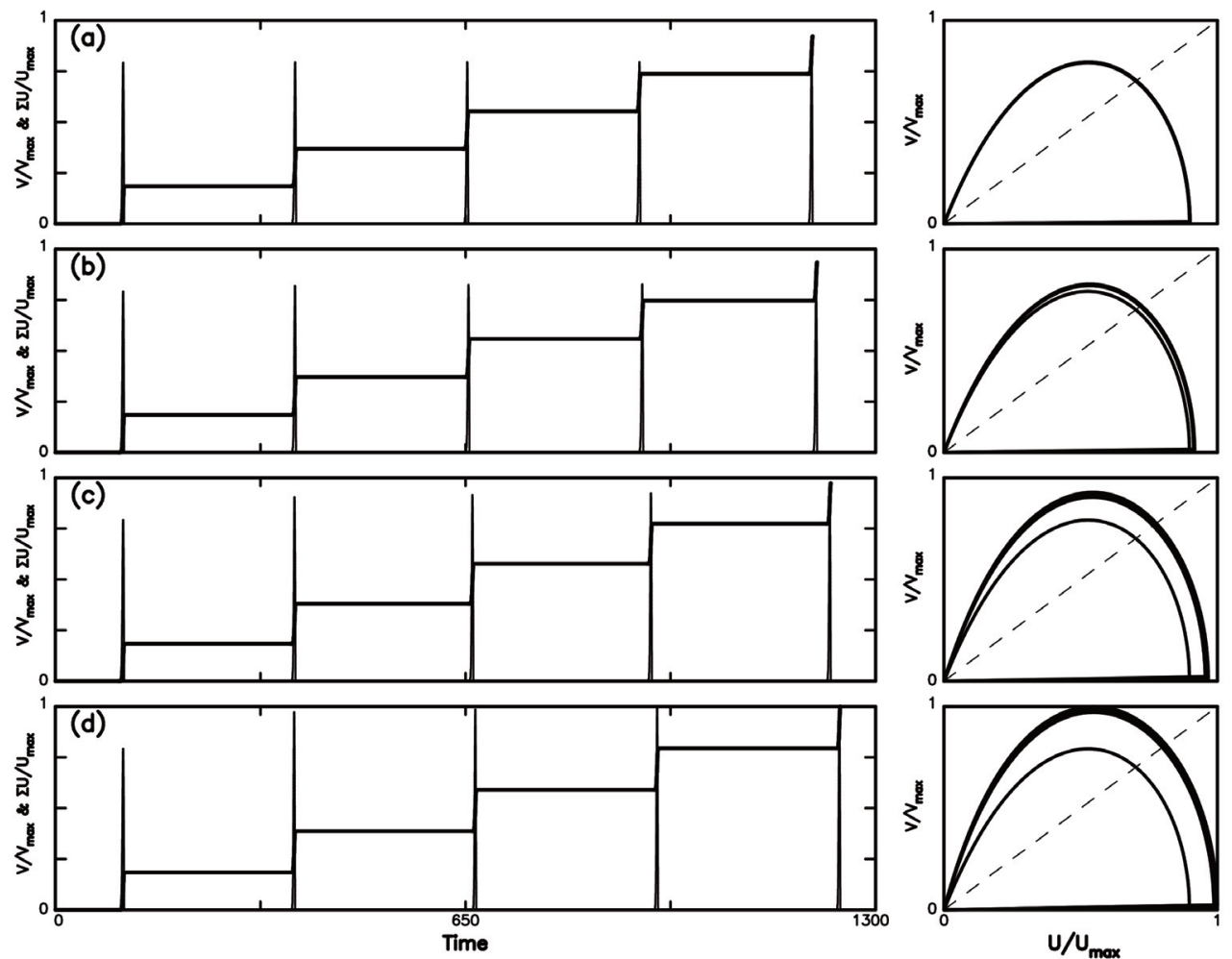

Figure 6. The time variations in $V / V_{\max }$ (thin solid line) and cumulative slip $\Sigma U / U_{\max }$ (solid line) and the phase portrait of $V / V_{\max }$ versus $U / U_{\max }$ (solid line) for four values of $B$ : (a) for $B=0.001$; (b) for $B=0.010$; (c) for $B=0.050$; and (d) for $B=0.100$ when $U_{c o}=0.3$. 
Jeen-Hwa Wang
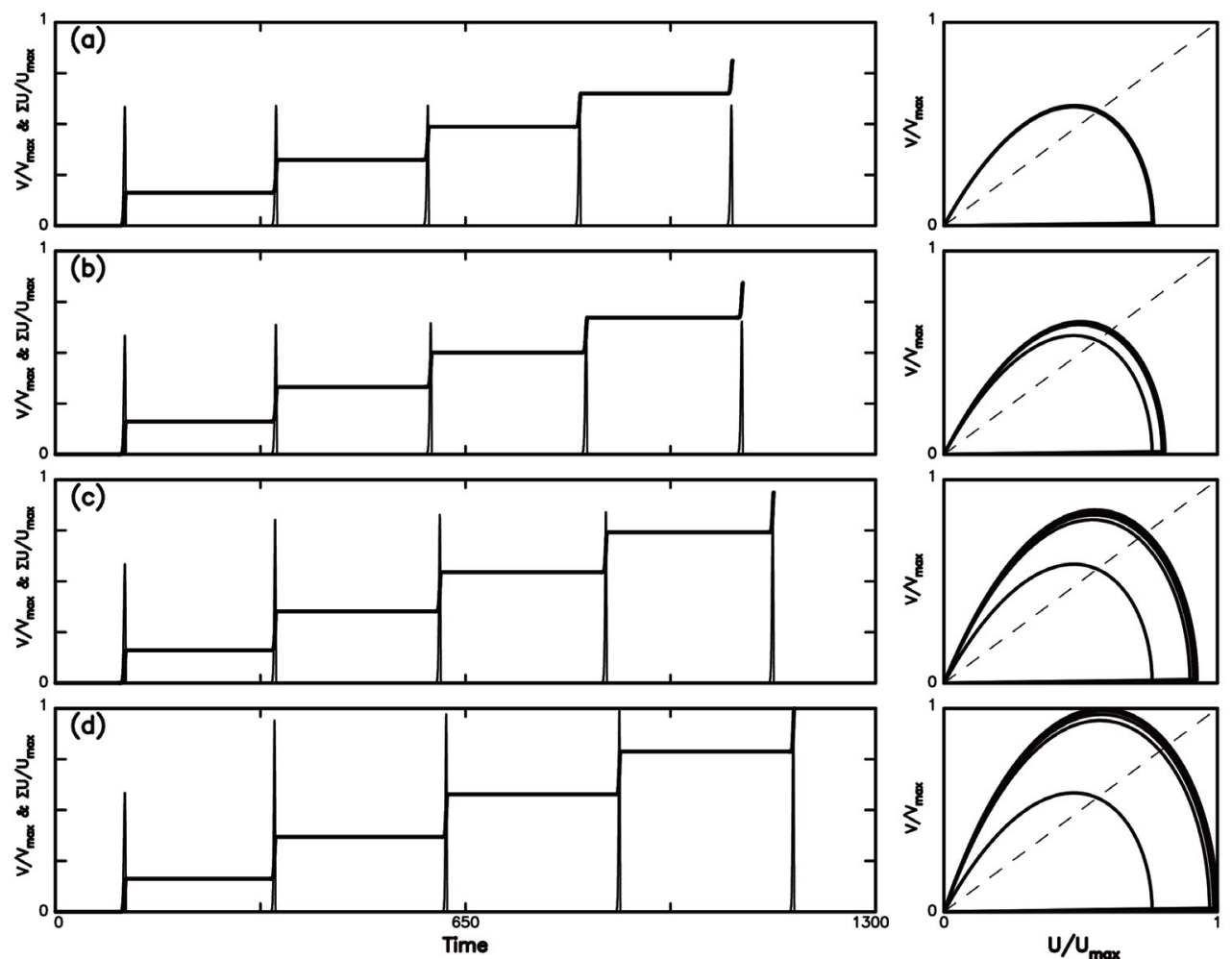

Figure 7. The time variations in $V / V_{\max }$ (thin solid line) and cumulative slip $\Sigma U / U_{\max }$ (solid line) and the phase portrait of $V / V_{\max }$ versus $U / U_{\max }$ (solid line) for four values of $B$ : (a) for $B=0.001$; (b) for $B=0.010$; (c) for $B=0.050$; and (d) for $B=0.100$ when $U_{c o}=0.5$.
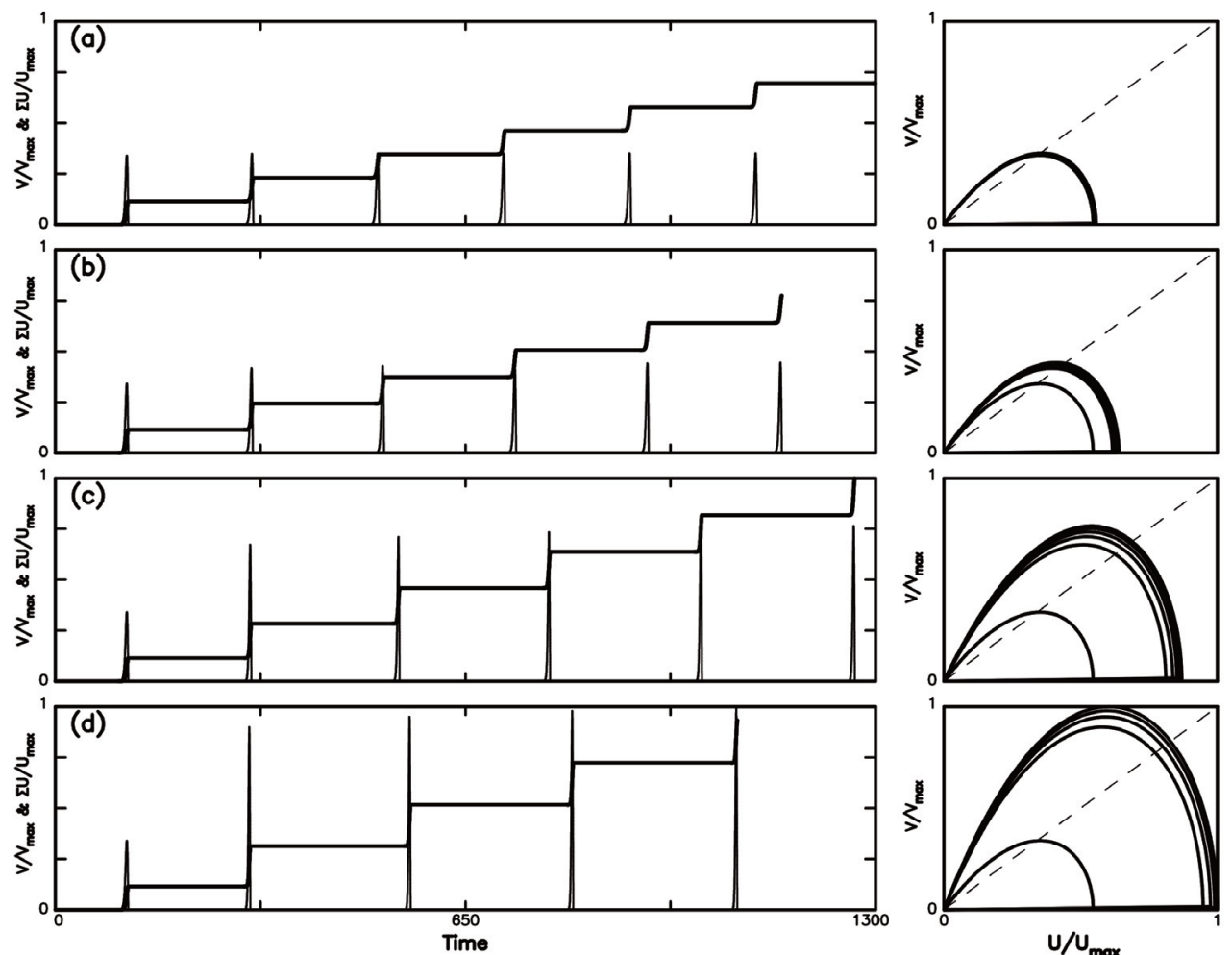

Figure 8. The time variations in $V / V_{\max }$ (thin solid line) and cumulative slip $\Sigma U / U_{\max }$ (solid line) and the phase portrait of $V / V_{\max }$ versus $U / U_{\max }$ (solid line) for four values of $B$ : (a) for $B=0.001$; (b) for $B=0.010$; (c) for $B=0.050$; and (d) for $B=0.100$ when $U_{c o}=0.7$. 
Static Friction and Earthquake Recurrence
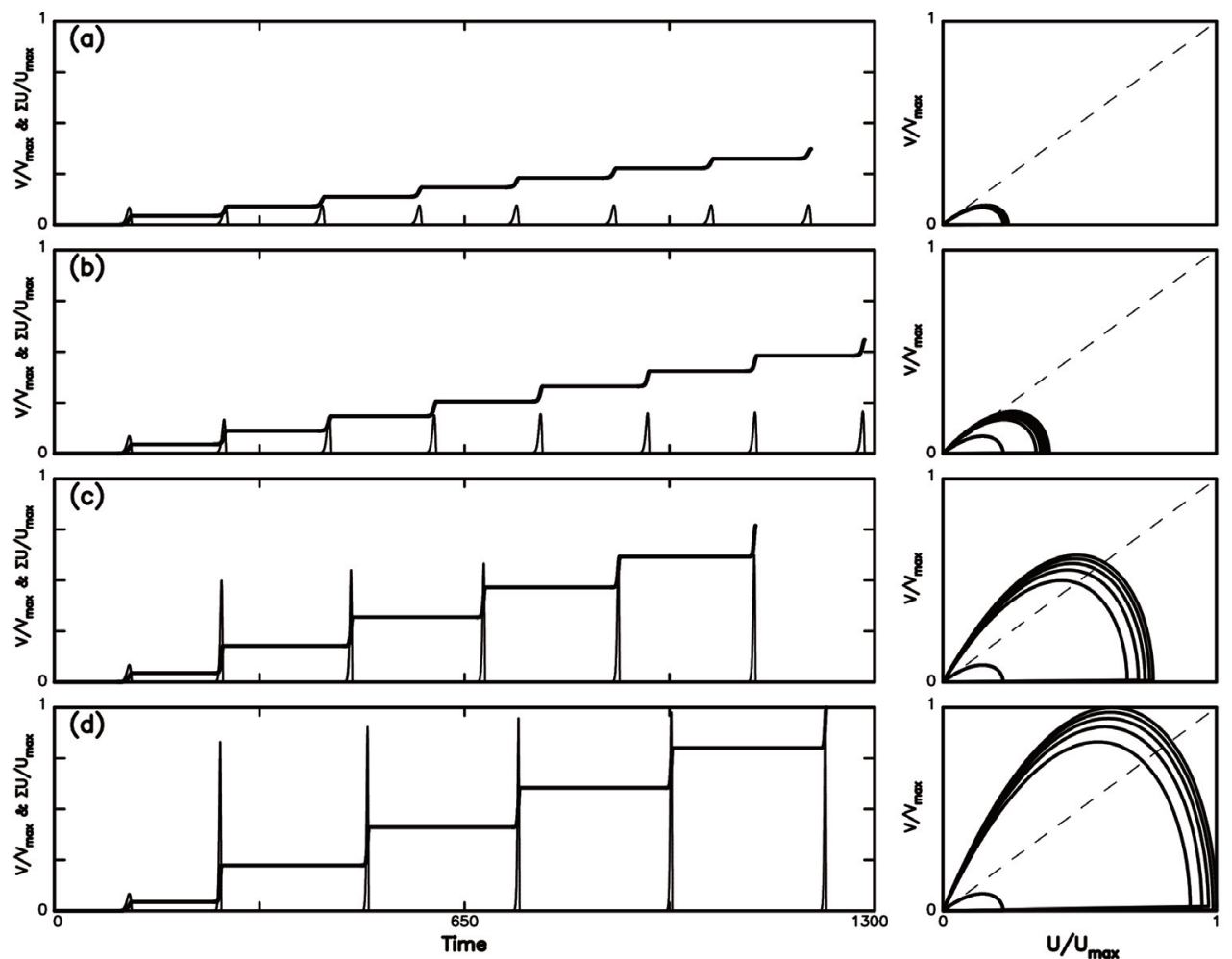

Figure 9. The time variations in $V / V_{\max }$ (thin solid line) and cumulative slip $\Sigma U / U_{\max }$ (solid line) and the phase portrait of $V / V_{\max }$ versus $U / U_{\max }$ (solid line) for four values of $B$ : (a) for $B=0.001$; (b) for $B=0.010$; (c) for $B=0.050$; and (d) for $B=0.100$ when $U_{c o}=0.9$.

\section{Discussion}

The left-handed-side panels of Figures 5-9 show the variations in velocities and displacements, the values of $V_{m}$, $D, \tau_{D}$, and $T_{R}$ are all similar for four values of $B$, i.e., $0.001,0.01,0.05$, and 0.1 , when $U_{c o}<0.3$ and different when $U_{c o} \geq 0.3$ These figures also display repetitive occurrences of simulation earthquakes. The patterns of earthquake recurrence depend on the values of model parameters. Meanwhile, the number of events increases with decreasing $B$ and slightly increases with $U_{c o}$. For the interseismic time $T_{R}$ in general increases with $B$ especially for $U_{c o}>0.3$. This means that a higher increasing rate of $\mu_{f}$ with time results in a longer interseismic time especially for $U_{c o}>0.3$. The earthquake recurrence is almost periodic when $U_{c o}$ is small; while the degree of aperiodicity increases when either $U_{c o}$ or $B$ increases. The three simple phenomenological models of earthquake recurrence proposed by Shimazaki and Nakata [1980] can approximately interpret the simulation results with smaller $U_{c o}$ and smaller $B$, yet not for those with larger $U_{c o}$ and larger $B$. For the present model, the critical friction force (related to the stress level, $\sigma_{c}$, for failure) is not constant and increases with time; while the base friction force (associated with the base stress level, $\sigma_{b}$ ) also increases with time. The two phenomena are particularly remarkable when $U_{c o}>0.3$. Since non-constant $\sigma_{c}$ and non-constant $\sigma_{o}$ cannot meet the stress conditions proposed by Shimazaki and Nakata [1980], their phenomenological models cannot interpret the present simulations results especially for $U_{c o}>0.3$.

On the other hand, Wang [2018] assumed that $U_{c}$ varies with cumulative slip in the following way: $U_{c}=U_{c o}+C \sum U$. Comparisons between Figures 5-9 of this study and Figures 8-12 shown in Wang [2018] show that the timestrengthening static friction coefficient produces a different effect on earthquake recurrence from the timewidening slip zone due to the wear process. The value of increasing rate of $T_{R}$ from time-widening $h$ is larger than that of decreasing rate from time-strengthening $\mu_{f}$. In other words, the effect on earthquake recurrence is stronger from time-widening $h$ than from the time-strengthening $\mu_{f}$. This can be seen from the formula: $U_{c}=\rho_{f} C_{V} h / \mu_{f} D_{o} \Lambda$ in which an increase in $h$ results in an increase in $U_{c}$ while an increase in $\mu_{f}$ leads to a decrease in $U_{c}$. Obviously an increase in $U_{c}$ with time is larger for $h$ from Wang [2018] than from $\mu_{f}$ in this study. Consequently the two model parameters $h$ and $\mu_{f}$ produce opposite effects on earthquake recurrence. For the model proposed by Wang [2018], the 


\section{Jeen-Hwa Wang}

critical friction force (related to the stress level, $\sigma_{c}$, for failure) is constant because of constant $\mu_{f}$ and the base friction force (associated with the base stress level, $\sigma_{b}$ ) also decreases with time. Hence, the time-predictable model proposed by Shimazaki and Nakata [1980] seems able to interpret the simulation results in Wang [2018] when the viscous effect is not included.

Figures 5-9 also reveal that $V_{m}$ slightly increases with $B$ when $U_{c o}<0.3$ and clearly increases with $B$ when $U_{c o} \geq 0.3$. The $D$ increases with $B$ and decreases with increasing $U_{c 0}$. The value of $D$ is an integral of velocity function from $\tau=0$ to $\tau=\tau_{D}$ as displayed in Figure 1. Figures 5-9 reveal that $\tau_{D}$ slightly decreases with increasing $B$ and increases with $U_{c o}$. In other words, smaller $B$ and larger $U_{c o}$ produce longer duration time of an earthquake. This produces the results that $D$ increases with $B$ and decreases with increasing $U_{c o}$. Larger $V_{m}$ and $D$ are associated with larger events. Hence, a higher increasing rate of $\mu_{f}$ with time together with a smaller value of $U_{c o}$ can produce a larger-sized earthquake. This phenomenon is particularly remarkable when $U_{c o}>0.3$. Figure 4 displays that and smaller $U_{c}$ due to smaller $B$ result in a higher force drop, $\mu F$, and lower static friction. Hence, higher $\Delta F$ or lower $\mu_{f}$ is more easily to produce a larger event and to lead to a perfectly periodic earthquake recurrence than lower $\Delta F$ or higher $\mu_{f}$. This phenomenon is particularly clear for smaller $U_{c o}$.

The right-handed-side panels of Figures 5-9 exhibit that the phase portraits are similar when $B<0.01$ and different when $B \geq 0.01$ for various values of $U_{c o}$ even though the patterns of their variations in $V$ and $U$ are similar. The size of a phase portrait increases with $B$. This reflects increases in both $T_{R}$ and $D$ of events with $B$. The absolute values of slope at non-zero fixed point are higher than 1 and slightly increase with time when $B \geq 0.010$. This suggests that the non-zero fixed points for all cases in study are not always an attractor. In addition, the zero fixed points are not an attractor, because the absolute values of slope at them are always higher than 1 .

An interesting question arises: Can the time-widening slip zone change the static friction coefficient on a fault? This might be possible because the wear process causing the time-widening slip zone seems able to increase the content of gouge inside the fault zone, thus changing the static friction coefficient. Of course, further studies should be conducted to answer this question.

\section{Summary}

Considering the effect due to time-strengthening static frictional coefficient, we assume $\mu_{f}(t)=1+B \times \log (t)$ and thus $U_{c}=U_{c o} /(1+B \times \log (t))$. Simulation results exhibit that $V_{m}, D, \tau_{D}$, and $T_{R}$ are all similar for five values of $U_{c o}$ when $B<0.01$ and different when $B \geq 0.01$. In general $V_{m}, D$, and $T_{R}$ increase with $B$; and $\tau_{D}$ slightly decreases with increasing $B$. An increase in $D$ is particularly remarkable when $U_{c 0}>0.3$. The earthquake recurrence is periodic when $U_{c o}$ or $B$ is small; while the degree of periodicity decreases when either $U_{c o}$ or $B$ increases. The three simple models of earthquake recurrence proposed by Shimazaki and Nakata [1980] can approximately interpret the simulation results with small $U_{c o}$ and small $B$, yet not for those with large $U_{c o}$ and large $B$. Simulation results exhibit that the time-strengthening static friction coefficient and the time-widening slip zone produce opposite effects on earthquake recurrence.

Acknowledgments. The author thanks an anonymous reviewer and Dr. Andrea Bizzarri (Associated Editor of the journal) for useful comments and suggestions to improve the article. The study was financially supported by Central Weather Bureau (Grant No.: MOTC-CWB-108-E-02) and Academia Sinica, Taiwan.

\section{References}

Ando, M. (1975). Source mechanisms and tectonic significance of historic earthquakes along the Nankai trough, Japan. Tectonpohys., 27, 119-140.

Bakun, W.H. and T.V. McEvilly (1984). Recurrence models and Parkfield, California, earthquakes. J. Geophys. Res., 89, 3051-3058.

Belardinelli, M.E. and E. Belardinelli (1996). The quasi-static approximation of the spring-slider motion. Nonl. Processes Geophys., 3, 143-149. 
Bizzarri, A. (2009). What does control earthquake ruptures and dynamic faulting? A review of different competing mechanism. Pure. Appl. Geophys., 166, 741-776.

Bizzarri, A. (2012a). Modeling repeated slip failures on faults governed by slip-weakening friction. Bull. Seism. Soc. Am., 102(2), 812-821, doi:10.1785/0120110141.

Bizzarri, A. (2012b). What can physical source models tell us about the recurrence time of earthquakes? Earth-Sci. Rev., 115, 304-318, http://dx.doi.org/10.1016/j.earscirev.2012.10.004.

Burridge, R. and L. Knopoff (1967). Model and theoretical seismicity. Bull. Seism. Soc. Am., 57, 341-371.

Cohen, S. (1979). Numerical and laboratory simulation of fault motion and earthquake occurrence. Rev. Geophys. Space Phys. 17(1), 61-72.

Franović, I., S. Kostić, M. Perc, V. Klinshov, V. Nekorkin, and J. Kurths (2016). Phase response curves for models of earthquake fault dynamics. Chaos, 26, 063105, http://dx.doi.org/10.1063/1.4953471.

Hirose, T. and M. Bystricky (2007). Extreme dynamic weakening of faults during dehydration by coseismic shear heating. Geophys. Res. Letts., 34, L14311 doi:10. 1029/2007GL030049.

Hudson, J.A. (1980). The excitation and propagation of elastic waves. Cambridge Monographs on Mechanics and Applied Mathematics, Cambridge Univ. Press, 226pp.

Kamer, S.L. and C. Marone (1998). The effect of shear load on frictional healing in simulated fault gouge. Geophys. Res. Letts., 25(24), 4561-4564.

Kittel, C., W.D. Knight, and M.A. Ruderman (1968). Mechanics. Berkeley Physics Course Vol. 1, McGraw-Hill Book Co., New York, 480pp.

Marone, C. (1998). Laboratory-derived friction laws and their application to seismic faulting. Ann. Rev. Earth Planet. Sci., 26, 643-669.

Marone, C., C.B. Rayleigh, and R.W. Scholz (1990). Frictional behavior and constitutive modeling of simulated fault gouge. J. Geophys. Res., 95(B5), 7007-7025.

Murray, J. and P. Segall (2002). Testing time-predictable earthquake recurrence by direct measurement of strain accumulation and release. Nature, 49, 287-291.

Nur, A. (1978). Nonuniform friction as a physical basis for earthquake mechanics. Pure. Appl. Geophys., 116, 964989.

Ohnaka, M. (1996). Nonuniformity of the constitutive law parameters for shear ruptures and quasistatic nucleation to dynamic rupture: A physical model of earthquake generation processes. Proc. Natl. Acad. Sci., 93, 37953802.

Press, WH., B.P. Flannery, S.A. Teukolsky, and W.T. Vetterling (1986). Numerical Recipes, Cambridge Univ. Press Cambridge, 818pp.

Reid, H.F. (1910). The California earthquake of April 18, 1906. In: Report of the State Investigation Commission 2, Mechanics of the Earthquake, Carnegie Inst., Washington, D.C.

Rice, J.R. (2006). Heating and weakening of faults during earthquake slip. J. Geophys. Res., 111, B05311, doi:10.1029/2005JB004006.

Rubinstein, J.L., W.L. Ellsworth, N.M., Beeler, B.D., Kilgore, D.A., Lockner, and H.M. Savage (2012). Fixed recurrence and slip models better predict earthquake behavior than the time- and slip-predictable models: 2 . Laboratory earthquakes. J. Geophys. Res. 117, B02307, doi:10.1029/2011JB008723.

Schwartz, D.P. and K.S. Coppersmith (1984). Fault behavior and characteristic earthquakes: examples from the Wasatch and San Andreas fault zones. J. Geophys. Res., 89, 5681-5698.

Shimamoto, T. and J.M. Logan (1981). Effects of simulated clay gouges on the sliding behavior of Tennessee sandstone. Tectonophys., 75, 243-255.

Shimazaki, K. and T. Nakata (1980). Time-predictable model for large earthquakes. Geophys. Res. Letts., 7, 279-282, doi.org/10.1029/GL007i004p00279.

Sibson, R.H. (1992). Implications of fault-valve behavior for rupture nucleation and recurrence. Tectonophys., 211, 283-293.

Sykes, L.R. and R.C. Quittmeyer (1981). Repeat times of great earthquakes along simple plate boundaries. In: Earthquake Prediction-An International Review, Maurice Ewing Series 4, AGU, Washington, D.C., USA, 217-247.

Thompson., J.M.T. and H.B. Stewart (1986). Nonlinear Dynamics and Chaos. John Wiley and Sons, New York, 376pp.

Turcotte, D.L. and G. Schubert (1982). GEODYNAMICS - Applications of Continuum Physics to Geological Problems, Wiley, 450pp. 


\section{Jeen-Hwa Wang}

Wang, J.H. (2005). Earthquakes rupturing the Chelungpu fault in Taiwan are time-predictable. Geophys. Res. Lett., 32, L06316 doi:10.1029/2004GL021884.

Wang, J.H. (2007). A dynamic study of the frictional and viscous effects on earthquake rupture: a case study of the 1999 Chi-Chi earthquake, Taiwan. Bull. Seism. Soc. Am., 97(4), 1233-1244.

Wang, J.H. (2009). Effect of thermal pressurization on the radiation efficiency. Bull. Seism. Soc. Am., 99(4), 22932304.

Wang, J.H. (2011). Thermal and pore fluid pressure history on the Chelungpu fault at a depth of 1111 meters during the 1999 Chi-Chi, Taiwan, earthquake. J. Geophys. Res., 116, B03302 doi:10.1029/2010JB007765.

Wang, J.H. (2016). Slip of a one-body spring-slider model in the presence of slip-weakening friction and viscosity. Ann. Geophys., 59(5), S0541, DOI:10. 4401/ag-7063.

Wang, J.H. (2017a). Slip of a two-degree-of-freedom spring-slider model in the presence of slip-weakening friction and viscosity. Ann. Geophys., 60(6), S0659, doi:10. 4401/ag-7295.

Wang, J.H. (2017b). Frictional and viscous effects on the nucleation phase of an earthquake nucleation. J. Seismol., 21(6), 1517-1539.

Wang, J.H. (2018). A study of earthquake recurrence based on a one-body spring-slider model in the presence of thermal-pressurized slip-weakening friction and viscosity. Nat. Hazards Earth Syst. Sci., 18, 1969-1983, https://doi.org/10.5194/nhess-18-1-2018.

Wang, J.H. (2019). A review on studies of the 1999 Chi-Chi earthquake for resolving the debatable problems in earthquake physics. Terr. Atmos. Ocean. Sci., 30(6), 1-18. 